\title{
Difficulty in identification of the frontal language area in patients with dominant frontal gliomas that involve the pars triangularis
}

\author{
Taiichi Saito, MD, PhD, ${ }^{1,4}$ Yoshihiro Muragaki, MD, PhD, ${ }^{1,2,4}$ Takashi Maruyama, MD, PhD, ,2,4 \\ Manabu Tamura, MD, PhD, ,2,4 Masayuki Nitta, MD, PhD, ${ }^{1,2,4}$ Shunsuke Tsuzuki, MD, PhD, ${ }^{1}$ \\ Yoshiyuki Konishi, PhD, ${ }^{2}$ Kotoe Kamata, MD, PhD, ${ }^{3}$ Ryuta Kinno, MD, PhD, ${ }^{4-6}$ \\ Kuniyoshi L. Sakai, PhD, ${ }^{4,6}$ Hiroshi Iseki, MD, PhD, ${ }^{1,2}$ and Takakazu Kawamata, MD, PhD ${ }^{1}$
}

Departments of ${ }^{1}$ Neurosurgery and ${ }^{3}$ Anesthesiology, ${ }^{2}$ Faculty of Advanced Techno-Surgery, Tokyo Women's Medical University; ${ }^{4}$ CREST, Japan Science and Technology Agency, Tokyo; ${ }^{6}$ Department of Basic Science, Graduate School of Arts and Sciences, University of Tokyo; and ${ }^{5}$ Division of Neurology, Department of Internal Medicine, Showa University Northern Yokohama Hospital, Kanagawa, Japan

OBJECTIVE Identification of language areas using functional brain mapping is sometimes impossible using current methods but essential to preserve language function in patients with gliomas located within or near the frontal language area (FLA). However, the factors that influence the failure to detect language areas have not been elucidated. The present study evaluated the difficulty in identifying the FLA in dominant-side frontal gliomas that involve the pars triangularis (PT) to determine the factors that influenced failed positive language mapping.

METHODS Awake craniotomy was performed on 301 patients from April 2000 to October 2013 at Tokyo Women's Medical University. Recurrent cases were excluded, and patients were also excluded if motor mapping indicated their glioma was in or around the motor area on the dominant or nondominant side. Eighty-two consecutive cases of primary frontal glioma on the dominant side were analyzed for the present study. MRI was used for all patients to evaluate whether tumors involved the PT and to perform language functional mapping with a bipolar electrical stimulator. Eighteen of 82 patients (mean age $39 \pm 13$ years) had tumors that showed involvement of the $P T$, and the detailed characteristics of these 18 patients were examined.

RESULTS The FLA could not be identified with intraoperative brain mapping in 14 (17\%) of 82 patients; 11 (79\%) of these 14 patients had a tumor involving the PT. The negative response rate in language mapping was only $5 \%$ in patients without involvement of the PT, whereas this rate was $61 \%$ in patients with involvement of the PT. Univariate analyses showed no significant correlation between identification of the FLA and sex, age, histology, or WHO grade. However, failure to identify the FLA was significantly correlated with involvement of the PT $(p<0.0001)$. Similarly, multivariate analyses with the logistic regression model showed that only involvement of the PT was significantly correlated with failure to identify the FLA $(p<0.0001)$. In 18 patients whose tumors involved the PT, only 1 patient had mild preoperative dysphasia. One week after surgery, language function worsened in $4(22 \%)$ of 18 patients. Six months after surgery, 1 $(5.6 \%)$ of 18 patients had a persistent mild speech deficit. The mean extent of resection was $90 \% \pm 7.1 \%$.

CONCLUSIONS Identification of the FLA can be difficult in patients with frontal gliomas on the dominant side that involve the PT, but the positive mapping rate of the FLA was $95 \%$ in patients without involvement of the PT. These findings are useful for establishing a positive mapping strategy for patients undergoing awake craniotomy for the treatment of frontal gliomas on the dominant side. Thoroughly positive language mapping with subcortical electrical stimulation should be performed in patients without involvement of the PT. More careful continuous neurological monitoring combined with subcortical electrical stimulation is needed when removing dominant-side frontal gliomas that involve the PT. http://thejns.org/doi/abs/10.3171/2015.8.JNS151204

KEY WORDS awake craniotomy; language function; glioma; pars triangularis; brain mapping; diagnostic and operative techniques 
$\mathrm{R}$ ESECTION of frontal gliomas on the dominant side that are located within or in close proximity to the frontal language area (FLA) as found by cortical stimulation in the frontal lobe represents a significant challenge. To minimize the risk of permanent postoperative speech dysfunction, detailed localization of the FLA is critical. The most effective and precise method is direct functional brain mapping using electrical stimulation during an awake craniotomy. ${ }^{8,18,24,25,33,34}$ However, we sometimes encounter patients whose eloquent language area cannot be located with intraoperative mapping. Sanai et al. reported a series of 250 patients who underwent intraoperative language mapping and stated that the language sites in only $58 \%$ of patients were successfully located..$^{33}$ The authors proposed that a tailored craniotomy permits gliomas to be aggressively resected if stimulation with an intensity of $6 \mathrm{~mA}$ (using a bipolar electrode, $60 \mathrm{~Hz}$, square wave) elicits no speech arrest or delay. This technique is called a "negative mapping strategy." Surprisingly, they reported that only $1.6 \%$ of surviving patients had a persistent language deficit. However, this strategy was employed in an experienced facility. A negative response in language mapping does not fully prevent postoperative persistent deterioration of speech function, which is observed after surgery in $9 \%-14.3 \%$ of such cases. ${ }^{5,18}$ Of note, a "negative response" means "attempted positive mapping has failed."

We postulated that the use of a "positive mapping strategy," meaning that the tumor is removed after identifying the language areas with a large craniotomy, is ideal and recommended. This is especially true in less experienced facilities because detecting the language area allows the surgeon to plan the resection area with more confidence..$^{30}$ Therefore, determining the factors that influence the failure to detect language areas is important. However, little has been reported about these factors. We empirically speculated that the tumor location is associated with a result of a negative response in language mapping and that identification of the FLA in particular can be difficult with frontal gliomas on the dominant side that involve the pars triangularis (PT). The PT is bordered superiorly by the inferior frontal sulcus, inferiorly by the anterior horizontal ramus, and caudally by the anterior ascending ramus and part of the posterior ramus of the sylvian fissure..$^{14}$ The PT cytoarchitectonically belongs to Broca's area and has been implicated in various aspects of linguistic functioning. We studied 82 consecutive patients with frontal gliomas on the dominant side who underwent an awake craniotomy and intraoperative cortical mapping to identify the FLA. The aim of the present study was to evaluate whether tumor location involving the PT was correlated with a negative response in language mapping to elucidate a factor that influences the failure of positive mapping.

\section{Methods}

\section{Patients and Preoperative Evaluation}

Awake craniotomy was performed in 301 patients from April 2000 to October 2013 at Tokyo Women's Medical University. Of these cases, we included 82 consecutive cases of primary frontal glioma on the dominant side. We excluded recurrent cases, and patients were also excluded if motor mapping indicated their glioma was in or around the motor area on the dominant or nondominant side. Eleven of the 82 patients were included from a previous study ${ }^{19}$ because the observation period of the present study overlapped with that of the previous one (from June 2007 to October 2009). An intracarotid amobarbital test revealed that the left cerebral hemisphere was language dominant in 79 of 82 patients, and the right side was dominant for language function in 3 patients. In all cases, the diagnosis was based on multimodal MRI, which included pre- and postcontrast T1-weighted, T2-weighted, FLAIR, and diffusion-weighted images. Using MR images, we evaluated whether the tumors involved the PT. We identified the PT as the area that was bordered superiorly by the inferior frontal sulcus, inferiorly by the anterior horizontal ramus, and caudally by the anterior ascending ramus and part of the posterior ramus of the sylvian fissure. Of 82 patients, the tumors in 18 patients ( 9 men and 9 women, mean age $39 \pm 13$ years) involved the PT, and we examined the detailed clinical characteristics of these 18 patients. The study protocol was approved by the Ethics Committee of the Tokyo Women's Medical University, and each patient provided informed consent before surgery.

\section{Surgery}

Surgery was performed according to the previously described concept of information-guided brain tumor removal, with the goal of maximal possible resection of the tumor with minimal risk of permanent postoperative neurological complications. ${ }^{22,23,35}$ Intraoperative MRI (AIRIS II; Hitachi Medical Corporation), updated neuronavigation, comprehensive neurophysiological monitoring, and detailed histopathological characterization of the resected tissue obtained at various stages of the procedure were used routinely. Surgery was generally performed with maximal possible removal of the contrast-enhanced area that was visualized on T1-weighted MRI for high-grade tumors and hyperintense areas on T2-weighted MRI for low-grade tumors. Histopathological diagnosis of tumors was based on the current criteria of the World Health Organization. $^{21}$

\section{Intraoperative Brain Mapping}

In all patients, tumor removal was performed during an awake craniotomy with a large bone flap and was guided by a "positive mapping strategy" of the cortical language areas and subcortical structures..$^{32}$ These procedures followed the established guidelines of the Japan Awake Surgery Conference. ${ }^{17}$ Prior to surgery, the patients were familiarized with the tasks used during intraoperative language mapping.

For intraoperative brain mapping, electrical stimulation of the cortex was applied with repetitive, square-wave, biphasic currents of alternating polarity (pulse width 0.2 msec, frequency $50 \mathrm{~Hz}$, duration 1-2 seconds) using an Ojemann cortical stimulator (OCS-1, Integra Radionics, Inc.) and a bipolar electrode with an interpolar distance of $5 \mathrm{~mm}$ and a tip diameter of $1 \mathrm{~mm}$. The stimulation was performed in a systematic manner, every 8 to $10 \mathrm{~mm}$ along the cortical surface. A continuous digital electrocorticogram was monitored to detect seizures and after- 
discharges. The stimulus intensity was increased steadily from $2 \mathrm{~mA}$ using stepwise increments of $1 \mathrm{~mA}$ until an effect was attained or abnormalities on electrocorticogram were noted. The maximum stimulus intensity was $8 \mathrm{~mA}$ (biphasic current $16 \mathrm{~mA}$ ).

For demonstration of the language assessment tasks to the patient, the dedicated intraoperative examination monitor for awake craniotomy ${ }^{38}$ was used, which allows realtime visualization, integration, and recording of a wide spectrum of data, including a view of the patient's face during the response, the type of test provided, the position of the cortical stimulator in the surgical field, etc. Cortical stimulation immediately preceded the task presentation for the patient and continued until the completion of each task presentation (1-2 seconds).$^{17}$ Language-related areas of the cerebral cortex were defined when their stimulation consistently interrupted, disturbed, or slowed the ability of the patient to name the pictured object, pronounce a familiar written Japanese word, and/or generate an action verb during demonstration of the picture task in the absence of seizures, after-discharges, and positive and negative motor responses of the tongue. To confirm reproducible results, each cortical area was stimulated at least 3 times, but never twice in succession. The eloquent cortical areas were delineated with a surgical pen upon completion of mapping. Removal of the tumor was accompanied by subcortical stimulation through the resection cavity and was intended to identify the language pathways. The devices used for this purpose and the parameters of stimulation, including the intensity, were similar to those used for cortical mapping. ${ }^{17}$

During the entire procedure, while the patient was awake, constant monitoring of the patient's spontaneous speech was achieved through continuous conversation with a member of the treatment team who specialized in language functioning and who provided specific tasks for evaluation of recall, counting, fluency, and comprehension. ${ }^{34}$

\section{Postoperative Evaluation}

The extent of resection was assessed with MRI performed within 48 hours of surgery. For semiautomatic volumetry to evaluate the extent of resection in the present study, we used 3D Slicer 4.0, which is freely downloadable from the website http://www.slicer.org. ${ }^{13}$ In enhanced tumors, we calculated the extent of resection using the contrast-enhanced area, and we used hyperintense areas on T2-weighted images in nonenhanced tumors. The initial evaluation of speech function was performed after the patient awoke from anesthesia and subsequently on a daily basis until discharge from the hospital. Thereafter, all patients were followed regularly in the outpatient clinic by the attending neurosurgeon.

\section{Statistical Analysis}

Correlations between the presence of involvement of the PT and patient characteristics (sex, histology, and identification of the FLA) were calculated by using the chi-square test. Additionally, correlations between the presence of involvement of the PT and other patient characteristics (age and extent of resection) were calculated by using the independent-samples t-test. Associations between the identification of the FLA and patient characteristics (sex, age, histology, WHO grade, and involvement of the PT) were calculated by using the chi-square test. Multivariate logistic regression analysis was used to explore the relationships between identification of the FLA and these variables. Statistical significance was defined as $\mathrm{p}<0.05$.

\section{Results}

Awake craniotomy was successfully performed in all 82 patients with a primary frontal glioma on the dominant side. The tumors of 18 patients involved the PT, but those of 64 patients did not. The demographics of the patient population, histology, identification of the FLA, and mean extent of resection by volumetry are shown in Table 1. Of all patients, the FLA could not be identified with intraoperative brain mapping in 14 patients (17\%). Additionally, $11(79 \%)$ of these 14 patients had tumors that involved the PT. The negative response rate in language mapping was only $5 \%$ in patients without involvement of the PT (positive mapping rate was 95\%), and this rate was $61 \%$ in patients with involvement of the PT (positive mapping rate was 39\%). The locations of the FLA are shown in Fig. 1. The FLAs were not identified on the PT in patients with involvement of the PT and were most frequently detected on the pars opercularis (Fig. 1A and B). The FLAs of patients without involvement of the PT were frequently identified on the PT and the pars opercularis (Fig. 1C and D).

Univariate analyses showed no significant correlation between identification of the FLA and sex, age, histology, or WHO grade (Table 2), whereas failure to identify the FLA was significantly correlated with involvement of the

TABLE 1. Patient demographic characteristics, clinical data, and pathological findings

\begin{tabular}{lccc}
\hline \multicolumn{1}{c}{ Characteristic } & $\begin{array}{c}\text { Involvement } \\
\text { of PT }\end{array}$ & $\begin{array}{c}\text { Noninvolvement } \\
\text { of PT }\end{array}$ & p Value* \\
\hline No. of cases & 18 & 64 & \\
\hline M/F & $9: 9$ & $39: 25$ & 0.4077 \\
\hline Mean age in yrs (range) & $39(19-63)$ & $39(18-65)$ & 0.8789 \\
\hline Histology, $n$ & & & 0.6733 \\
\hline A & 1 & 11 & \\
\hline OA & 5 & 14 & \\
\hline O & 3 & 12 & \\
\hline AA & 2 & 4 & \\
\hline AOA & 3 & 5 & \\
\hline AO & 3 & 9 & \\
\hline GBM & 1 & 9 & 0.0001 \\
\hline Identification of FLA, $n(\%)$ & & & \\
\hline Yes & $7(39)$ & $61(95)$ & \\
\hline No & $11(61)$ & $3(5)$ & \\
\hline Mean extent of resection & $90 \pm 7.1$ & $86 \pm 16$ & \\
\pm SD (\%) & & & \\
\hline A & & & \\
\hline
\end{tabular}

$A=$ astrocytoma; $A A=$ anaplastic astrocytoma; $A O=$ anaplastic oligodendroglioma; $\mathrm{AOA}=$ anaplastic oligoastrocytoma; $\mathrm{GBM}=$ glioblastoma; $\mathrm{O}$ = oligodendroglioma; $\mathrm{OA}=$ oligoastrocytoma.

* Statistical analysis was performed using the chi-square test and independent-samples t-test. 
A

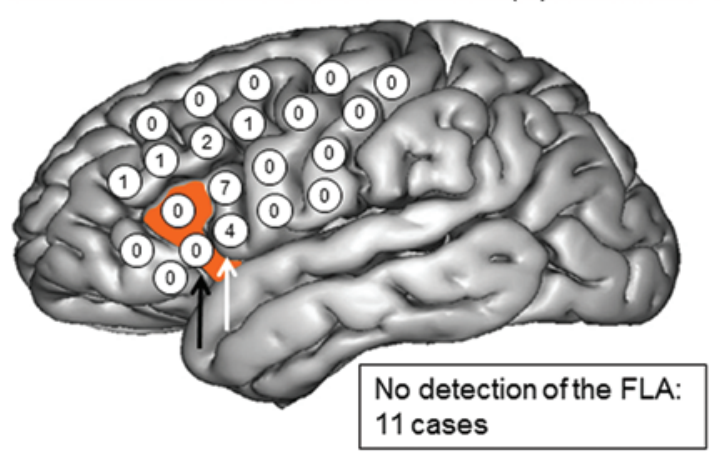

C

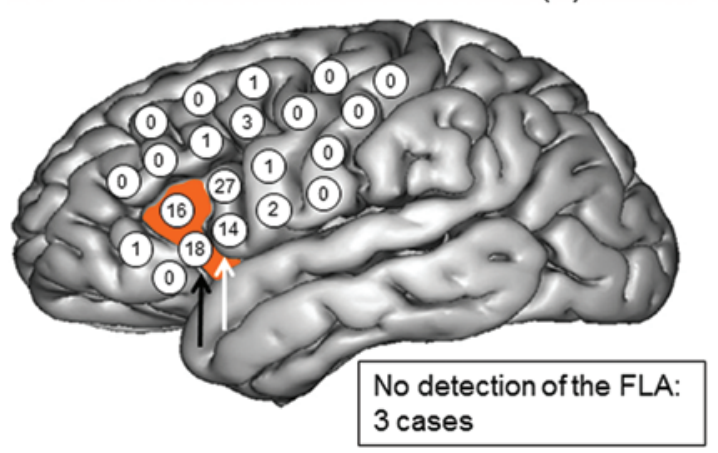

B

Involvement of the PT (\%: N/18)

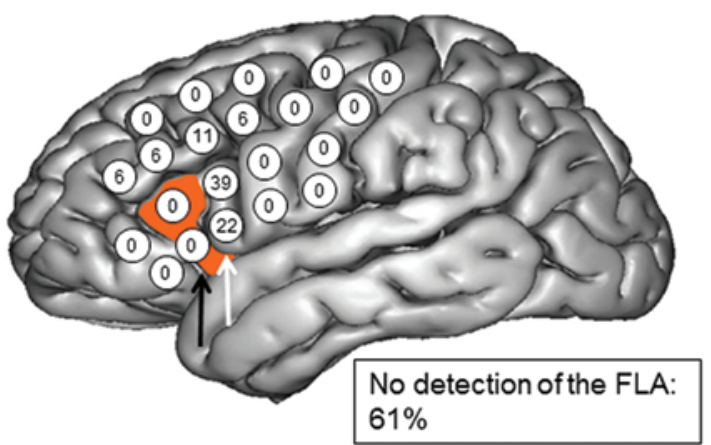

D Non-involvement of the PT (\%: N/64)

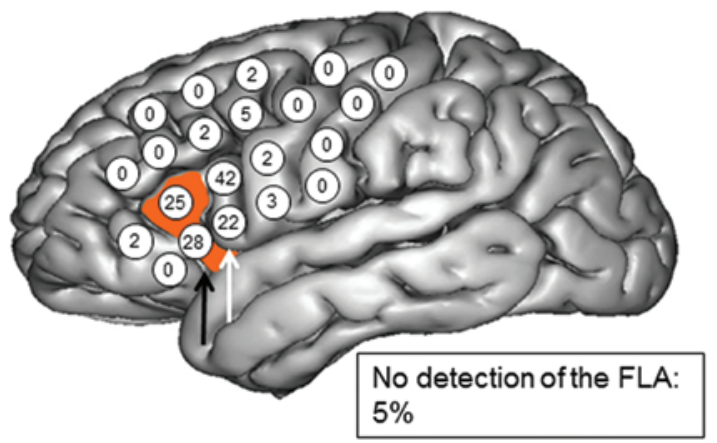

FIG. 1. Diagrams of the dominant side of the normalized hemisphere showing the location of identified FLAs in patients with and without involvement of the pars triangularis (PT). A: The total number of identified FLAs on which electrical stimulation induced speech dysfunction in 18 patients whose tumors involved the PT. The FLA could not be identified on the exposed brain cortex in 11 patients. B: The percentage of identified FLAs in all 18 patients whose tumors involved the PT. The FLA could not be identified on the exposed brain cortex in $11(61 \%)$ of 18 patients. C: The total number of identified FLAs on which electrical stimulation induced speech dysfunction in 64 patients whose tumors did not involve the PT. The FLA could not be identified on the exposed brain cortex in only 3 patients. D: The percentage of identified FLAs in all 64 patients whose tumors did not involve the PT. The FLA could not be identified on the exposed brain cortex in only $3(5 \%)$ of 64 patients. The black arrows indicate the anterior horizontal ramus of the sylvian fissure. The white arrows indicate the anterior ascending ramus of the sylvian fissure. The PT is shown in orange. Figure is available in color online only.

PT $(\mathrm{p}<0.0001)$. Similarly, multivariate analyses with the logistic regression model showed that only involvement of the PT was significantly correlated with failure to identify the FLA ( $p<0.0001)$ (Table 3). Consequently, identification of the FLA was significantly difficult in frontal gliomas on the dominant side that involved the PT.

Detailed clinical characteristics of the 18 patients with primary frontal gliomas on the dominant side are shown in Table 4. An intracarotid amobarbital test revealed that the left cerebral hemisphere was language dominant in 17 of 18 patients and that the right side was dominant for language function in only 1 patient (Case 18). Only 1 patient (Case 4) had mild dysphasia before surgery. In 11 (61\%) of 18 patients, the FLA could not be identified (negative response in language mapping). One week after surgery, language function worsened in $4(22 \%)$ of 18 patients. Six months after surgery, 1 (5.6\%) of 18 patients had a persistent mild speech deficit. The mean extent of resection by volumetry was $90 \% \pm 7.1 \%$.

\section{Representative Cases} Case 7

A 19-year-old woman presented with generalized seizures. She had no other neurological symptoms, and her preoperative speech function was normal. MRI showed that the tumor was located in the left insula and inferior frontal gyrus and involved the PT. The tumor had a hypointense signal and partial enhancement with contrast medium on T1-weighted imaging, and a hyperintense signal on T2-weighted imaging (Fig. 2A). An intracarotid amobarbital test revealed that the left cerebral hemisphere was language dominant. Tumor removal was performed during an awake craniotomy. Electrical stimulations for intraoperative brain mapping could not identify the FLA on the exposed brain cortex. We removed the tumor under the guidance of cortical and subcortical stimulation for language functional mapping with constant monitoring of the patient's spontaneous speech. No language dysfunction was noted during removal of the tumor. However, during removal of the deep part of the insular tumor, transcortical motor-evoked potentials decreased to $<60 \%$ of preremoval amplitude and spontaneous movement of the patient's right hand worsened slightly. Therefore, we stopped the resection. The patient had no language dysfunction immediately after surgery. Postoperative MRI revealed $90 \%$ resection of the tumor (Fig. 2B), and the histopathological diagnosis was an anaplastic oligodendroglioma. Her speech function was normal 1 week and 6 months after surgery. 
TABLE 2. Subgroups of FLA identification and patient characteristics

\begin{tabular}{cccc}
\hline & \multicolumn{2}{c}{ Identification of the FLA } & \\
\cline { 2 - 3 } Characteristic & Yes & No & p Value* \\
\hline No. of cases & 68 & 14 & \\
\hline Sex & & & \\
\hline M & 39 & 9 & 0.6294 \\
\hline F & 29 & 5 & \\
\hline Mean age in yrs & & & \\
\hline$\leq 39$ & 39 & 9 & 0.6294 \\
\hline$>39$ & 29 & 5 & \\
\hline Tumor histology & & & \\
\hline Astrocytic & 24 & 4 & 0.6250 \\
\hline Oligodendroglial & 44 & 10 & \\
\hline WHO grade & & & \\
\hline II & 38 & 7 & 0.1631 \\
\hline III & 20 & 7 & \\
\hline IV & 10 & 0 & \\
\hline Involvement of PT & & & \\
\hline Yes & 7 & 11 & \\
\hline No & 61 & 3 & \\
\hline
\end{tabular}

* Statistical analysis was performed using the chi-square test.

\section{Case 16}

A 32-year-old man experienced generalized seizures. He had no other neurological symptoms or signs, and his preoperative speech function was normal. MRI showed a tumor centered in the left inferior and middle frontal gyrus that involved the PT. The tumor had a hypointense signal, nonenhancement with contrast medium on T1-weighted imaging, and a hyperintense signal on T2-weighted imaging (Fig. 2C). An intracarotid amobarbital test revealed that the left cerebral hemisphere was language dominant. Tumor removal was performed during an awake craniotomy. Electrical stimulations for intraoperative brain mapping could not identify the FLA on the exposed brain cortex including the tumor surface. We removed the tumor under the guidance of cortical and subcortical stimulation for language functional mapping with constant monitoring of the patient's spontaneous speech. The area of speech arrest was recorded during the task of naming and verb generation $(4,6$, and $8 \mathrm{~mA}, 50 \mathrm{~Hz})$ with subcortical stimulation of the cavity after the anterior part of the tumor was extirpated. This area caused not only speech arrest but also perseveration in the verb generation task. During removal of the posterior part of the tumor, the patient demonstrated speech arrest, and subcortical stimulation (4 and $6 \mathrm{~mA}$ ) on the profound resection cavity elicited speech arrest. Therefore, we stopped additional resection. The patient had mild motor aphasia immediately after surgery. Postoperative MRI revealed $90 \%$ resection of the tumor (Fig. 2D), and the histopathological diagnosis was an oligoastrocytoma. His speech function recovered 1 week after surgery and was normal at 6 months after surgery.

\section{Case 18}

An intracranial mass lesion was incidentally discovered in a 25-year-old man. He had no neurological symptoms, and his preoperative speech function was normal. MRI showed that the tumor was mainly located in the corpus callosum and the right inferior frontal gyrus and involved the PT. The tumor had a hypointense signal, nonenhancement with contrast medium on T1-weighted imaging, and a hyperintense signal on T2-weighted imaging (Fig. 2E). An intracarotid amobarbital test revealed that the right cerebral hemisphere was language dominant. Tumor removal was performed during an awake craniotomy. Electrical stimulations for intraoperative brain mapping could not identify the FLA on the exposed brain cortex including the tumor surface. We removed the tumor under the guidance of cortical and subcortical stimulation for language functional mapping with constant monitoring of the patient's spontaneous speech. No speech arrest was noted during removal of the tumor. The tumor in the corpus callosum partially extended to the contralateral frontal lobe. To preserve higher brain function, we did not remove the contralaterally extended part of the tumor. The patient did not experience speech disorder immediately after surgery. Postoperative MRI revealed $95 \%$ resection of the tumor (Fig. 2F), and the histopathological diagnosis was an oligodendroglioma. His speech function was normal 1 week and 6 months after surgery.

\section{Discussion}

Results of the present study indicate that tumor location involving the PT is significantly correlated with a negative response in language mapping. The FLA could not be identified in only $3(5 \%)$ of 64 patients with a frontal glioma on the dominant side without involvement of the PT, whereas a negative response for the FLA was observed in $11(61 \%)$ of 18 patients with involvement of the PT. Several reports have demonstrated the incidence of negative cortical mapping for language areas. Sanai et al. reported

TABLE 3. Multivariate logistic regression model predicting identification of the FLA

\begin{tabular}{lccc}
\hline \multicolumn{1}{c}{ Regressor } & Coefficient & OR $(95 \% \mathrm{Cl})$ & $\mathrm{p} \mathrm{Value}^{*}$ \\
\hline Sex: F vs M & 0.735 & $2.085(0.358-12.153)$ & 0.4141 \\
\hline Age: $\leq 39$ yrs vs <39 yrs & 0.754 & $0.471(0.080-2.763)$ & 0.4039 \\
\hline Histology: astrocytic vs oligodendroglial & 0.338 & $0.713(0.126-4.038)$ & 0.7021 \\
\hline WHO grade: II vs III, IV & 0.124 & $1.131(0.235-5.437)$ & 0.8774 \\
\hline Involvement of the PT: yes vs no & 3.883 & $0.021(0.004-0.119)$ & $<0.0001$ \\
\hline OR = odds ratio
\end{tabular}

* Statistical analysis was performed using a logistic regression model test. 
TABLE 4. Summary of clinical characteristics in 18 patients with frontal glioma on the dominant side that involved the PT

\begin{tabular}{|c|c|c|c|c|c|c|c|c|}
\hline \multirow[b]{2}{*}{$\begin{array}{c}\text { Case } \\
\text { No. }\end{array}$} & \multirow[b]{2}{*}{$\begin{array}{l}\text { Age in } \\
\text { Yrs, Sex }\end{array}$} & \multirow{2}{*}{$\begin{array}{c}\text { Language } \\
\text { Dominant } \\
\text { Side }\end{array}$} & \multirow[b]{2}{*}{$\begin{array}{c}\text { Preop Language } \\
\text { Dysfunction }\end{array}$} & \multirow[b]{2}{*}{$\begin{array}{c}\text { Tumor } \\
\text { Histology }\end{array}$} & \multirow[b]{2}{*}{$\begin{array}{c}\text { Identification } \\
\text { of FLA }\end{array}$} & \multicolumn{2}{|c|}{ Postop Language Dysfunction } & \multirow[b]{2}{*}{$\begin{array}{l}\% \text { Lesion } \\
\text { Resection }\end{array}$} \\
\hline & & & & & & 1 Wk Postop & 6 Mos Postop & \\
\hline 1 & $30, \mathrm{M}$ & $\mathrm{Lt}$ & No & AA & No & No & No & 98 \\
\hline 2 & $39, \mathrm{M}$ & $\mathrm{Lt}$ & No & $\mathrm{AO}$ & No & No & No & 98 \\
\hline 3 & $57, \mathrm{~F}$ & $\mathrm{Lt}$ & No & 0 & No & Mild motor aphasia & No & 95 \\
\hline 4 & $29, \mathrm{M}$ & $\mathrm{Lt}$ & Mild motor dysphasia & $\mathrm{AO}$ & No & Motor aphasia, repetition error & Mild motor dysphasia & 90 \\
\hline 5 & $42, F$ & $\mathrm{Lt}$ & No & $\mathrm{OA}$ & No & No & No & 70 \\
\hline 6 & $62, \mathrm{M}$ & $\mathrm{Lt}$ & No & $\mathrm{AOA}$ & Yes & No & No & 95 \\
\hline 7 & $19, F$ & $\mathrm{Lt}$ & No & $\mathrm{AO}$ & No & No & No & 90 \\
\hline 8 & $43, F$ & $\mathrm{Lt}$ & No & $A$ & No & No & No & 90 \\
\hline 9 & $26, \mathrm{M}$ & $\mathrm{Lt}$ & No & 0 & Yes & No & No & 100 \\
\hline 10 & $41, F$ & $\mathrm{Lt}$ & No & GBM & Yes & No & No & 90 \\
\hline 11 & $33, \mathrm{M}$ & $\mathrm{Lt}$ & No & AA & No & No & No & 90 \\
\hline 12 & $63, F$ & $\mathrm{Lt}$ & No & $\mathrm{OA}$ & Yes & No & No & 85 \\
\hline 13 & $29, F$ & Lt & No & $\mathrm{AOA}$ & Yes & No & No & 85 \\
\hline 14 & $50, F$ & $\mathrm{Lt}$ & No & $\mathrm{OA}$ & Yes & Mild motor aphasia, paraphasia & No & 95 \\
\hline 15 & $38, \mathrm{M}$ & $\mathrm{Lt}$ & No & $\mathrm{OA}$ & No & Mild motor aphasia, paraphasia & No & 80 \\
\hline 16 & $32, \mathrm{M}$ & $\mathrm{Lt}$ & No & $\mathrm{OA}$ & No & No & No & 90 \\
\hline 17 & $52, F$ & $\mathrm{Lt}$ & No & $\mathrm{AOA}$ & Yes & No & No & 90 \\
\hline 18 & $25, \mathrm{M}$ & Rt & No & 0 & No & No & Mild motor dysphasia & 95 \\
\hline
\end{tabular}

a series of 250 patients who underwent language mapping during a tailored craniotomy (tumor plus adjacent areas) and a negative response for the language area in $42 \% .33$ Furthermore, in many patients (up to $40 \%$ ), frontal lobe speech arrest sites could not be identified in their series. In a series of 309 patients, Kim et al. failed to identify the motor speech areas in $105(39 \%)$ of 273 procedures during a tailored craniotomy. ${ }^{18}$ Recently, Chacko et al. described $21(31 \%)$ of 67 patients in whom eloquent areas were not identified. ${ }^{5}$ On the other hand, in a series of 60 patients, Duffau et al. described no cases of negative mapping including in 14 patients who underwent mapping for the speech center. ${ }^{11}$ In contrast to tailored craniotomy, as described above, they performed a large craniotomy with cortical stimulation to identify language sites. Compared with these reports, the tumors that involved the PT in the present study had a higher incidence of a negative response in language mapping.

The frontal operculum is a relevant language area in the dominant hemisphere. ${ }^{25}$ Several reports have demonstrated surgical treatment for gliomas in the frontal opercular region. ${ }^{2,25,27}$ Most recently, Rolston et al. analyzed 43 cases of gliomas involving the dominant side of the frontal operculum, ${ }^{27}$ where each tumor was classified as involving the pars orbitalis, PT, pars opercularis, or a combination of some or all of these areas. An awake craniotomy was performed in 32 patients of 43 patients, and 11 patients underwent tumor removal surgery under general anesthesia. From cortical mapping results, $9(56 \%)$ of 16 patients without postoperative deficits had no positive speech sites compared with $8(62 \%)$ of 13 patients with a postoperative language deficit in whom positive language areas could not be identified. Each rate (56\% and 62\%) is higher compared with the rate of a negative response in language mapping of $42 \%$ in their largest study, ${ }^{33}$ and was equivalent to our rate for frontal gliomas involving the PT. These results also suggest that tumor localization involving the frontal operculum is associated with a negative response in language mapping.

Brodmann's areas 45 (PT) and 44 (pars opercularis) cytoarchitectonically belong to Broca's area ${ }^{1}$ and are believed to be correlated with speech expression. However, several language cortical mapping studies during awake craniotomy in patients with glioma demonstrated a high degree of variety in frontal language eloquent sites, such as the lower region of the precentral gyrus, the pars orbitalis, and the dorsal premotor cortex. ${ }^{2,25,26}$ Additionally, several reports have indicated that patients with gliomas that involve the frontal operculum do not necessarily show preoperative language dysfunction. ${ }^{2,25,27}$ Duffau et al. have postulated that this mechanism of functional reshaping, called "brain plasticity," may be the result of the recruitment of perilesional areas or even the contralateral cortex. Such functional reorganization was demonstrated in several neurofunctional imaging $2-4,20,36$ and intraoperative cortical mapping studies. . $10,12,30,31$ On the other hand, our group previously reported the results of a preoperative functional MRI study with the picture-sentence matching task in 21 patients with left frontal glioma. ${ }^{19} \mathrm{We}$ aimed to show how normally existing syntax-related networks are functionally reorganized by a lesion. The patients were divided into 3 groups: patients with a glioma in the lateral premotor cortex $(\mathrm{n}=$ $7)$, opercular/triangular regions $(n=7)$, and other frontal regions $(n=7)$. Furthermore, we recruited 7 normal agematched participants for functional MRI experiments. The modulation of activation in the other frontal regions group was exactly replicated in the normal group. In contrast, we found dramatic changes in the activation patterns in the 

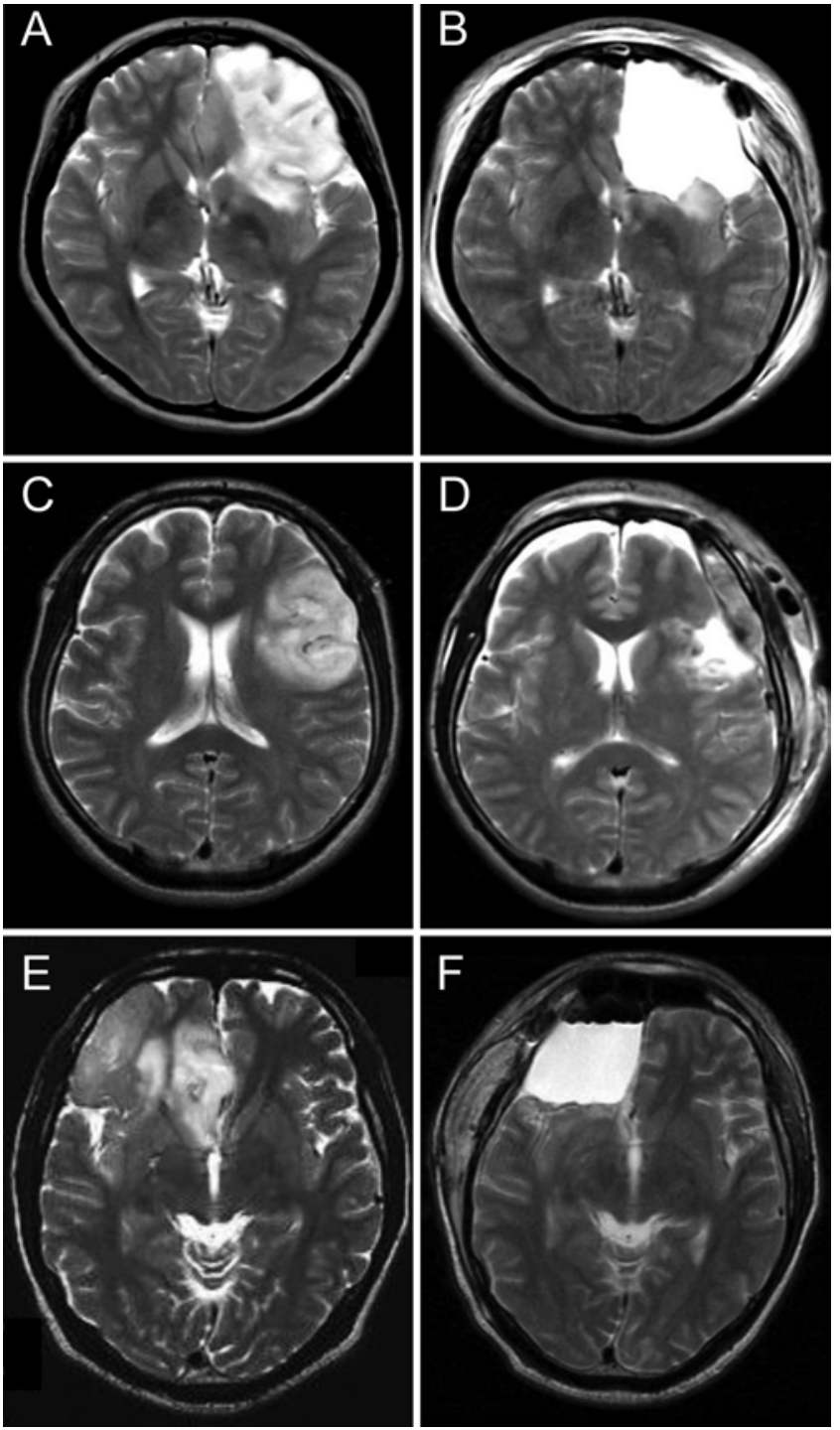

FIG. 2. Axial MR images obtained in 3 representative cases involving the PT. A and B: Case 7. MR images obtained in a 19-year-old woman with left frontal lobe anaplastic oligodendroglioma. Preoperative T2weighted MR image (A) showing a tumor in the left frontal lobe that did not involve the PT. Electrical stimulation for intraoperative brain mapping could not identify the FLA. Postoperative T2-weighted MR image (B) showing $90 \%$ resection of the tumor. C and D: Case 16. MR images obtained in a 32-year-old man with left frontal lobe oligoastrocytoma. Preoperative T2-weighted MR image $(C)$ showing a tumor in the left inferior and middle frontal gyrus that involved the PT. Electrical stimulation for intraoperative brain mapping could not identify the FLA. Postoperative T2-weighted MR image (D) showing $90 \%$ resection of the tumor. $\mathbf{E}$ and F: Case 18. MR images obtained in a 25 -year-old man with right frontal lobe oligodendroglioma. Preoperative T2-weighted MR image (E) showing a tumor in the corpus callosum and right frontal lobe that involved the PT. An intracarotid amobarbital test revealed that the right cerebral hemisphere is language dominant. Electrical stimulations for intraoperative brain mapping could not identify the FLA. Postoperative T2-weighted MR image (F) showing 95\% resection of the tumor.

opercular/triangular regions and lateral premotor cortex groups, which were accompanied by abnormal overactivity and/or underactivity in the syntax-related regions. Strikingly, in the opercular/triangular regions group, activation was absent in all left frontal regions. These data may be related to the results of the negative response in language mapping in the patients with left frontal gliomas that involved the PT in the present study.

Why is the rate of a negative response in language mapping different depending on the involvement of the PT? In the present study, the FLA was identified in all 14 patients whose tumor involved the pars opercularis but not the PT (data not shown). Cytoarchitectonic differences in neuronal densities have also been reported in the frontal operculum. Based on cytoarchitectonic differences, Chawluk et al. suggested that the pars opercularis is more suited to unimodal motor processing, such as articulation. However, the PT receives modality-specific input and then performs cross-modal associations, which are required for performing complex linguistic functions. ${ }^{6}$ Furthermore, in 3 righthanded patients with epilepsy, Sahin et al. investigated neuronal activity using intracranial electrophysiology by recording local field potentials from populations of neurons using depth electrodes implanted in language-related brain regions while patients read words (nouns and verbs) verbatim or grammatically inflected them (present/past, singular/plural). They found that neighboring probes within the PT revealed distinct neuronal activity for lexical (approximately $200 \mathrm{msec}$ ), grammatical (approximately $320 \mathrm{msec}$ ), and phonological (approximately $450 \mathrm{msec}$ ) processing. These findings suggest that the PT plays an important role in the functional language network..$^{29}$ Therefore, we assume that drastic functional language reorganization (neuroplasticity) occurs when a tumor invades the PT. Neuroplasticity is conceivable only in a dynamic, not static, view of brain organization. ${ }^{9,10}$ We speculate that neuroplasticity proceeds in stages and that scattered incomplete functional language relocalization occurs before neuroplasticity is completed. Stimulation with a bipolar probe creates an electrical field in which the current density is homogenous, and the electric field lines between both tips are nearly parallel. ${ }^{34}$ Therefore, in the present study, scattered localized language areas may not have been detected with the limited bipolar electrical stimulation performed in the 11 patients with tumors that involved the PT. Additionally, a cortical response is produced by a charge density that is sufficient to trigger action potentials in the affected tissue. We applied a maximum stimulus intensity of $8 \mathrm{~mA}$ (biphasic current $16 \mathrm{~mA}$ ) during awake craniotomy, and this intensity may be insufficient to trigger action potentials. Strategies to increase the applied charge density have improved the sensitivity. However, with increasing current density, the potential for tissue injury increases.$^{16}$ Given these considerations, one possibility is that 11 of the 18 patients with a frontal glioma on the dominant side that involved the PT had incomplete FLA reorganization with scattered localization, whereas the FLAs of the other 7 patients had been sufficiently reorganized.

We used a "positive mapping strategy" with a large craniotomy, similar to the strategy of Duffau's group. ${ }^{7}$ Indeed, the rate of a negative response in language mapping was only $5 \%$ in patients without involvement of the PT in the present study. However, Sanai et al. reported that tailored craniotomies, even without localization of positive language sites, permit gliomas to be aggressively resected (negative mapping strategy). ${ }^{33}$ Similarly, Kim et al. pro- 
posed that a negative response in eloquent areas provides a safe margin for resection, with a lower incidence of postoperative neurological deficits. ${ }^{18}$ However, one should bear in mind that this strategy was employed in an experienced facility. A negative response in language mapping does not fully prevent persistent postoperative deterioration of speech function. ${ }^{5,18}$ More recently, Chacko et al. described a series of 67 patients who underwent awake craniotomy and reported that 14 patients $(20.8 \%)$ failed to elicit electrophysiological responses despite the fact that the tumors were located near the eloquent area. ${ }^{5}$ In this group, 3 patients (21\%) developed intraoperative deficits. We believe that the use of the "positive mapping strategy" has a lower risk of permanent language deficits and is recommended in less experienced facilities. In the present study, we found that identification of the FLA can be difficult in frontal gliomas on the dominant side that involved the PT. However, 1 week after surgery, language function worsened in 4 patients (22\%), and only 1 patient $(5.6 \%)$ had a persistent mild speech deficit in this group. Our findings are very useful for the "positive mapping strategy" during an awake craniotomy in patients with frontal gliomas on the dominant side. Considering these findings, we should thoroughly perform language cortical mapping in patients whose tumors do not involve the PT, and use the "positive mapping strategy" including subcortical electrical stimulation to preserve language function. More careful continuous neurological monitoring combined with subcortical electrical stimulation is needed when removing frontal gliomas on the dominant side that involve the PT in patients in whom the FLA cannot be identified. This is because the majority of persistent neurological deficits after awake craniotomy for glioma removal are related to injury to eloquent subcortical structure ${ }^{37}$ that can be identified with intraoperative electrical stimulation. When a continuous language deficit is observed or subcortical stimulation elicits a reproducible, intraoperative speech arrest, we should stop additional resection to avoid persistent language dysfunction.

The present study has some limitations. First, although a preoperative functional MRI study is useful for identification of the FLA with high sensitivity and specificity, ${ }^{15,28}$ we did not perform this study in all patients. Therefore, we observed no direct correlation between brain cortical mapping results and activated sites on functional MRI. Further investigation aiming to directly correlate image findings with brain mapping results will strengthen our hypothesis that identification of the FLA can be difficult in frontal gliomas on the dominant side that involve the PT. Second, our study was conducted at a single institution and was retrospective. Furthermore, a clear reason for the absence of positive language sites in patients with tumors that involve the PT has not been identified. Our observations need to be reproduced and validated. Therefore, additional prospective and multiinstitutional studies including neurofunctional imaging analyses are needed.

\section{Conclusions}

We demonstrated that identification of the FLA can be difficult in frontal gliomas on the dominant side that involve the PT. Conversely, the positive mapping rate of the
FLA was 95\% in patients with tumors that did not involve the PT. Our findings are useful for the "positive mapping strategy" during an awake craniotomy in patients with frontal gliomas on the dominant side. Given these findings, we should thoroughly perform language cortical mapping and use the "positive mapping strategy" in patients whose tumors do not involve the PT. More careful continuous neurological monitoring combined with subcortical electrical stimulation is needed when removing frontal gliomas on the dominant side that involve the PT in patients in whom the FLA cannot be identified. Further prospective studies combining neurofunctional imaging analyses such as functional MRI are essential to fully evaluate the reorganization of language function in patients with frontal gliomas on the dominant side that involve the PT.

\section{Acknowledgments}

This study was supported by grants from the Japan National Cancer Research and Development Fund and Core Research for Evolutionary Science and Technology (CREST), Japan Science and Technology Agency, and Japan Agency for Medical Research and Development (AMED). We extend special thanks to Drs. Tomokatsu Hori, Yoshikazu Okada, Kintomo Takakura, Takashi Komori, Osami Kubo, and Katsuya Maebayashi, Ms. Soko Ikuta, Satoko Fukuchi, Rieko Ishihara, and Mr. Takashi Sakayori for valuable supervision and help with clinical work and data analysis.

\section{References}

1. Amunts K, Schleicher A, Bürgel U, Mohlberg H, Uylings $\mathrm{HB}$, Zilles K: Broca's region revisited: cytoarchitecture and intersubject variability. J Comp Neurol 412:319-341, 1999

2. Benzagmout M, Gatignol P, Duffau H: Resection of World Health Organization Grade II gliomas involving Broca's area: methodological and functional considerations. Neurosurgery 61:741-753, 2007

3. Bizzi A, Nava S, Ferrè F, Castelli G, Aquino D, Ciaraffa F, et al: Aphasia induced by gliomas growing in the ventrolateral frontal region: assessment with diffusion MR tractography, functional MR imaging and neuropsychology. Cortex 48:255-272, 2012

4. Briganti C, Sestieri C, Mattei PA, Esposito R, Galzio RJ, Tartaro A, et al: Reorganization of functional connectivity of the language network in patients with brain gliomas. AJNR Am J Neuroradiol 33:1983-1990, 2012

5. Chacko AG, Thomas SG, Babu KS, Daniel RT, Chacko G, Prabhu K, et al: Awake craniotomy and electrophysiological mapping for eloquent area tumours. Clin Neurol Neurosurg 115:329-334, 2013

6. Chawluk JB, Mesulam MM, Hurtig H, Kushner M, Weintraub S, Saykin A, et al: Slowly progressive aphasia without generalized dementia: studies with positron emission tomography. Ann Neurol 19:68-74, 1986

7. De Benedictis A, Moritz-Gasser S, Duffau H: Awake mapping optimizes the extent of resection for low-grade gliomas in eloquent areas. Neurosurgery 66:1074-1084, 2010

8. Duffau H: Contribution of cortical and subcortical electrostimulation in brain glioma surgery: methodological and functional considerations. Neurophysiol Clin 37:373-382, 2007

9. Duffau H: The huge plastic potential of adult brain and the role of connectomics: new insights provided by serial mappings in glioma surgery. Cortex 58:325-337, 2014

10. Duffau H: Lessons from brain mapping in surgery for lowgrade glioma: insights into associations between tumour and brain plasticity. Lancet Neurol 4:476-486, 2005

11. Duffau H, Capelle L, Sichez J, Faillot T, Abdennour L, Law 
Koune JD, et al: Intra-operative direct electrical stimulations of the central nervous system: the Salpetrière experience with 60 patients. Acta Neurochir (Wien) 141:1157-1167, 1999

12. Duffau H, Denvil D, Capelle L: Long term reshaping of language, sensory, and motor maps after glioma resection: a new parameter to integrate in the surgical strategy. $\mathbf{J}$ Neurol Neurosurg Psychiatry 72:511-516, 2002

13. Egger J, Kapur T, Fedorov A, Pieper S, Miller JV, Veeraraghavan $\mathrm{H}$, et al: GBM volumetry using the 3D Slicer medical image computing platform. Sci Rep 3:1364, 2013

14. Foundas AL, Leonard CM, Gilmore RL, Fennell EB, Heilman KM: Pars triangularis asymmetry and language dominance. Proc Natl Acad Sci U S A 93:719-722, 1996

15. Giussani C, Roux FE, Ojemann J, Sganzerla EP, Pirillo D, Papagno C: Is preoperative functional magnetic resonance imaging reliable for language areas mapping in brain tumor surgery? Review of language functional magnetic resonance imaging and direct cortical stimulation correlation studies. Neurosurgery 66:113-120, 2010

16. Gordon B, Lesser RP, Rance NE, Hart J Jr, Webber R, Uematsu S, et al: Parameters for direct cortical electrical stimulation in the human: histopathologic confirmation. Electroencephalogr Clin Neurophysiol 75:371-377, 1990

17. Kayama T: The guidelines for awake craniotomy guidelines committee of the Japan awake surgery conference. Neurol Med Chir (Tokyo) 52:119-141, 2012

18. Kim SS, McCutcheon IE, Suki D, Weinberg JS, Sawaya R, Lang FF, et al: Awake craniotomy for brain tumors near eloquent cortex: correlation of intraoperative cortical mapping with neurological outcomes in 309 consecutive patients. Neurosurgery 64:836-845, 345-346, 2009

19. Kinno R, Ohta S, Muragaki Y, Maruyama T, Sakai KL: Differential reorganization of three syntax-related networks induced by a left frontal glioma. Brain 137:1193-1212, 2014

20. Kośla K, Pfajfer L, Bryszewski B, Jaskólski D, Stefańczyk L, Majos A: Functional rearrangement of language areas in patients with tumors of the central nervous system using functional magnetic resonance imaging. Pol J Radiol 77:39-45, 2012

21. Louis DN, Ohgaki H, Wiestler OD, Cavenee WK, Burger PC, Jouvet A, et al: The 2007 WHO classification of tumours of the central nervous system. Acta Neuropathol 114:97-109, 2007

22. Muragaki Y, Iseki H, Maruyama T, Kawamata T, Yamane F, Nakamura R, et al: Usefulness of intraoperative magnetic resonance imaging for glioma surgery. Acta Neurochir Suppl 98:67-75, 2006

23. Muragaki Y, Iseki H, Maruyama T, Tanaka M, Shinohara C, Suzuki T, et al: Information-guided surgical management of gliomas using low-field-strength intraoperative MRI. Acta Neurochir Suppl 109:67-72, 2011

24. Nossek E, Matot I, Shahar T, Barzilai O, Rapoport Y, Gonen $\mathrm{T}$, et al: Failed awake craniotomy: a retrospective analysis in 424 patients undergoing craniotomy for brain tumor. J Neurosurg 118:243-249, 2013

25. Peraud A, Ilmberger J, Reulen HJ: Surgical resection of gliomas WHO grade II and III located in the opercular region. Acta Neurochir (Wien) 146:9-18, 2004

26. Quiñones-Hinojosa A, Ojemann SG, Sanai N, Dillon WP, Berger MS: Preoperative correlation of intraoperative cortical mapping with magnetic resonance imaging landmarks to predict localization of the Broca area. J Neurosurg 99:311-318, 2003

27. Rolston JD, Englot DJ, Benet A, Li J, Cha S, Berger MS: Frontal operculum gliomas: language outcome following resection. J Neurosurg 122:725-734, 2015

28. Roux FE, Boulanouar K, Lotterie JA, Mejdoubi M, LeSage JP, Berry I: Language functional magnetic resonance imaging in preoperative assessment of language areas: correlation with direct cortical stimulation. Neurosurgery 52:13351347,2003

29. Sahin NT, Pinker S, Cash SS, Schomer D, Halgren E: Sequential processing of lexical, grammatical, and phonological information within Broca's area. Science 326:445-449, 2009

30. Saito T, Muragaki Y, Maruyama T, Tamura M, Nitta M, Okada Y: Intraoperative functional mapping and monitoring during glioma surgery. Neurol Med Chir (Tokyo) 55:1-13, 2015

31. Saito T, Muragaki Y, Miura I, Tamura M, Maruyama T, Nitta $\mathrm{M}$, et al: Functional plasticity of language confirmed with intraoperative electrical stimulations and updated neuronavigation: case report of low-grade glioma of the left inferior frontal gyrus. Neurol Med Chir (Tokyo) 54:587-592, 2014

32. Saito T, Tamura M, Muragaki Y, Maruyama T, Kubota Y, Fukuchi S, et al: Intraoperative cortico-cortical evoked potentials for the evaluation of language function during brain tumor resection: initial experience with 13 cases. J Neurosurg 121:827-838, 2014

33. Sanai N, Mirzadeh Z, Berger MS: Functional outcome after language mapping for glioma resection. $\mathbf{N}$ Engl J Med 358:18-27, 2008

34. Szelényi A, Bello L, Duffau H, Fava E, Feigl GC, Galanda $\mathrm{M}$, et al: Intraoperative electrical stimulation in awake craniotomy: methodological aspects of current practice. Neurosurg Focus 28(2):E7, 2010

35. Tamura M, Muragaki Y, Saito T, Maruyama T, Nitta M, Tsuzuki S, et al: Strategy of surgical resection for glioma based on intraoperative functional mapping and monitoring. Neurol Med Chir (Tokyo) 55:383-398, 2015

36. Tate MC, Herbet G, Moritz-Gasser S, Tate JE, Duffau H: Probabilistic map of critical functional regions of the human cerebral cortex: Broca's area revisited. Brain 137:27732782, 2014

37. Trinh VT, Fahim DK, Shah K, Tummala S, McCutcheon IE, Sawaya R, et al: Subcortical injury is an independent predictor of worsening neurological deficits following awake craniotomy procedures. Neurosurgery 72:160-169, 2013

38. Yoshimitsu K, Suzuki T, Muragaki Y, Chernov M, Iseki H: Development of modified intraoperative examination monitor for awake surgery (IEMAS) system for awake craniotomy during brain tumor resection. Conf Proc IEEE Eng Med Biol Soc 2010:6050-6053, 2010

\section{Disclosures}

The authors report no conflict of interest concerning the materials or methods used in this study or the findings specified in this paper.

\section{Author Contributions}

Conception and design: Muragaki, Saito, Iseki. Acquisition of data: Saito, Maruyama, Tamura, Nitta, Tsuzuki, Konishi, Kamata, Kinno, Sakai. Analysis and interpretation of data: Muragaki, Saito, Tamura, Kinno, Sakai. Drafting the article: Saito. Critically revising the article: Muragaki, Kinno, Kawamata. Reviewed submitted version of manuscript: Saito, Maruyama, Tamura, Nitta, Tsuzuki, Konishi, Kamata, Kinno, Sakai, Iseki, Kawamata. Approved the final version of the manuscript on behalf of all authors: Muragaki. Statistical analysis: Saito. Administrative/ technical/material support: Maruyama, Tamura, Nitta, Tsuzuki, Konishi, Kamata, Kinno, Sakai, Iseki. Study supervision: Muragaki, Kawamata.

\section{Correspondence}

Yoshihiro Muragaki, Faculty of Advanced Techno-Surgery, Institute of Advanced Biomedical Engineering and Science, Tokyo Women's Medical University, 8-1 Kawada-cho, Shinjuku-ku, Tokyo 162-8666, Japan. email: ymuragaki@twmu.ac.jp. 